\title{
Patterns of Young Children's Number Sense Development as Assessed by How Many Hidden Game
}

\author{
Connie Cassy Ompok ${ }^{1 *}$, Brian Doig ${ }^{2}$ and Maria Tambagas ${ }^{3}$ \\ ${ }^{1,3}$ Universiti Malaysia Sabah, Kota Kinabalu, Sabah, Malaysia \\ ${ }^{2}$ Deakin University, Geelong VIC 3220, Australia
}

\begin{abstract}
Using games as a context for assessing children's mathematical abilities is not unusual, but this study investigated the potential of games designed specifically for the purpose of assessment. Children in prior-to-school settings in Sabah, Malaysian Borneo, were exposed to a range of such games, including the how many hidden game, which focuses on number sense, which is the subject of this paper. Children's responses while playing the game were recorded, and we report the range of successful responses to the game, as well as the age of the children involved.
\end{abstract}

Keywords: How many hidden; assessment; mathematical abilities; number sense; game

Copyright: This is an open access article distributed under the terms of the Creative Commons Attribution-NonCommercialShareAlike 4.0 International (CC BY-NC-SA 4.0) license which permits unrestricted use, distribution, and reproduction in any medium, for non-commercial purposes, provided the original work is properly cited.

\section{INTRODUCTION}

The data for this paper are drawn from a larger study of the usefulness of a gamesbased approach for assessing children's abilities in mathematics. The focus of this paper is on the how many hidden game, which has a focus on number sense. This game originated in Australia and known as Gumnut

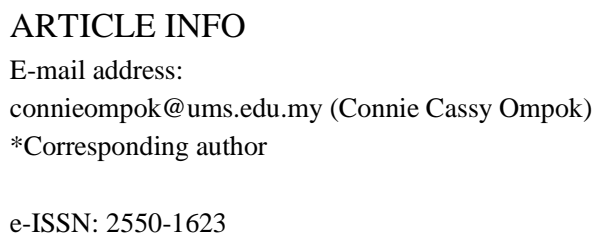

Manuscript received: 26 Aug 2018; Accepted: 1 Oct 2018. Date of publication: 10 Dec 2018 game, and used readily available nuts from eucalyptus trees as playing pieces. However, researchers in Malaysian Borneo modify this game and name it as How many hidden. Any small objects can be used, and in Sabah, plastic counters were used as the playing pieces. The development of children's prior-toschool number sense is important, as Aunola et al., (2004), Claessens et al., (2009), Romano et al., (2010), have shown that number sense proficiency is beneficial to a child's school mathematics learning. Davydov (1976) too, in his work on quantities, and their relationships, while eschewing number, points out that the basic relationships between quantities is a foundation for problem solving in the later years of children's mathematics learning. 
This paper outlines this innovative approach to assessing young children's informal mathematical abilities through the use of games designed as instruments for assessment through non-threatening environment.

\section{METHODOLOGY}

Altogether 74 children, aged 4 to 6 years, played the how many hidden game with one of their teachers. All children were not yet in formal schooling, and as had developed their quantity knowledge and understandings informally.

\section{Procedure}

Children were asked if they would like to play a game, the How many hidden Game, with one of their teachers. Those who agreed then played the game and the highest quantity for which they responded correctly was recorded as their 'score'. This does not mean, that a child who played with a maximum quantity of 5, knew all the possible combinations that make 5 (see Table 1 below for these). However, each child had the opportunity to repeat the game for that number.

\section{The How Many Hidden Game}

The How many hidden game is a game for a teacher, parent, or other numerate person, and a child. The game focuses on the child's ability to conceptualise a quantity, and its component (sub-quantities) parts. This is to say, the game is about playing with number sense, and allows an assessment of the child's number sense capabilities.

The How many hidden game develops practice in number sense. For example, the starting quantity might be 5 , and the game requires the child to provide one of the two sub-quantities, of 5, for all possible combinations. Additionally, only one of the sub-quantities is visible, so that physical counting is not available to aid the child's response. For the quantity 5 , this is represented as in Table 1 below.

Table 1: Sub-quantities of the quantity 5

\begin{tabular}{cc}
\hline $\begin{array}{c}\text { Visible sub- } \\
\text { quantity }\end{array}$ & $\begin{array}{c}\text { Hidden sub- } \\
\text { quantity }\end{array}$ \\
\hline 5 & 0 \\
4 & 1 \\
3 & 2 \\
2 & 3 \\
1 & 4 \\
0 & 5 \\
\hline
\end{tabular}

The visible sub-quantity lies in front of the child, while the hidden sub-quantity remains hidden from the child. Once the child has named the hidden sub-quantity, they get to see if they were correct or not, thus allowing them to see the previously hidden sub-quantity, and correct themselves if necessary.

Note that the hidden sub-quantity has to be recovered from an internalised splitting of the initial quantity (5 in the example above) in order to play the game successfully.

\section{RESULTS}

The children's scores (their highest game number) were grouped by age and described in three figures below, and by descriptive statistics. In order to make the data clearer in the figures, children's responses have been ordered from lowest to highest response number. The figures are presented below. 


\section{The How Many Hidden Games}

If the child is very young, try two counters to start, but experience suggest that three is an ideal starting point from two-year-olds and upwards.

\section{Part 1}

Show the stones (counters) to the child.

Ask: Can you tell me how many (counters) I have?

If the response is not two, ask them to count. If the child not agree that there are two counters, then either try one counter, or abandon this task.

\section{Part 2}

I am going to hide the (counters) in my hands.

Do this behind your back, or by turning away from the child. Place some (counters) in each hand. Show your closed hands to the child.

Ask: Which hand do you want to see (open)? Open that hand.

How many (counters) are there? Wait for their response.

How many (counters) are hidden? After the child's response, open that hand.

Were you correct?

Repeat the process, changing the number of (counters).

\section{Part 3}

Say: Now it is you turn. Don't let me see you hide the (counters).

After it is your turn to say how many in each hand, ask: Was I correct? How do you know?

\section{Part 4}

If the child is fluent, play again with 4 (counters), then 5 , until the task is too difficult.

\section{Part 5}

Play the game again, exactly as above, but without (counters). Say the following: I have some pretend (counters) in my hand. There are three of them. How many are there? If the child agrees with the number of counters, go back to Part 2 and continues as before. If the child does not 'see' the pretend (counters) then do not continue the game.
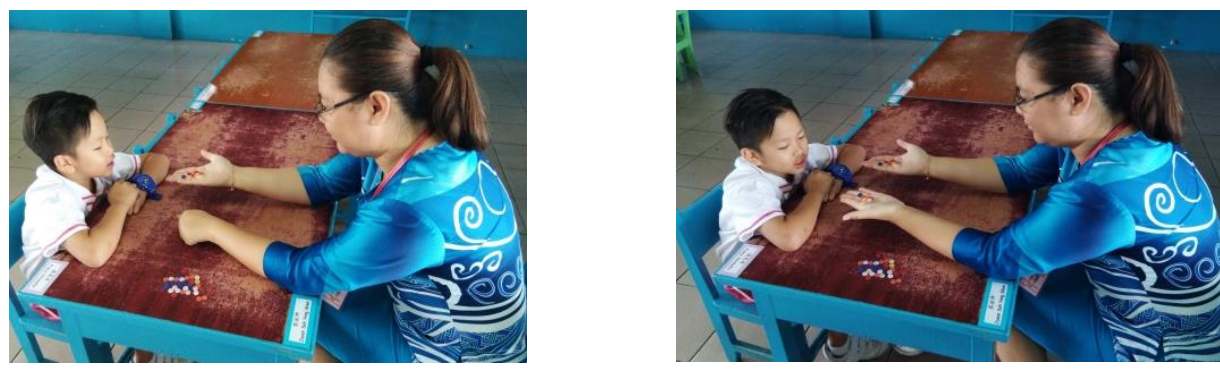

Figure 1 and 2: Playing How many hidden game 


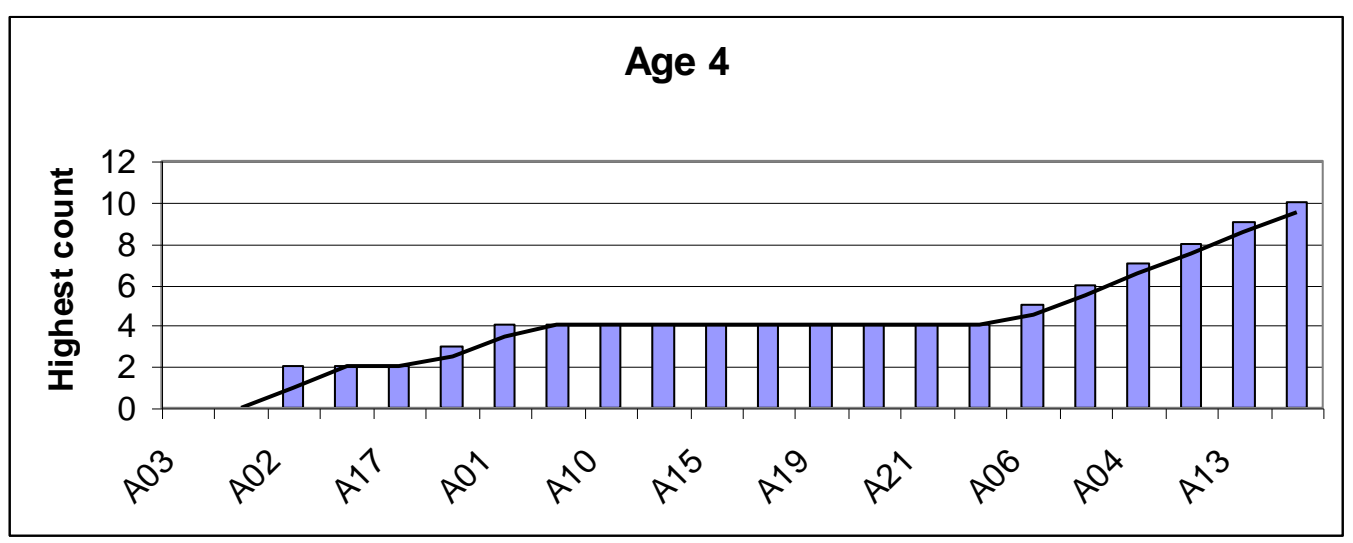

Figure 3: Four-year-olds' How many hidden game performances $(\mathrm{N}=22)$

Note, that in Figure 3, there appears to be a 'barrier' for many children, in the sample, at their age. However, we have found, shown in Figure 1, that this is not true for all children, as six children (27\%) were able to work with quantities beyond 5 , and one child was able to work with the quantity 10 . Anecdotal evidence appears to support that for the younger child, the quantity understanding is aligned with their age: that is, 2-year-olds are capable with a quantity at least 2, 3-year-olds with a quantity at least 3 , and so on. In our sample of 4-year-olds, 4 children (18\%) were only able to work with quantities less than 4 , while 10 children (45\%) could work with the quantity 4. Two children were unable to start the game, and were credited with a quantity of 0 . The mean quantity for the 4-year-old group was 4.27, with a Standard Deviation of 2.53 .

Figure 4 shows that, by the age of 5 years, the majority of our sample had broken free of any restriction to their age. The majority of children could work with quantities greater than 5 , some up to 10 , and a few beyond 10 . Only 4 of the 24 in the 5 -year-old sample $(16.7 \%)$ were working below 5, while 2 $(8.3 \%)$ were at a quantity of 5 , and of the remaining 18 children, 15 (62.5\%) were able to

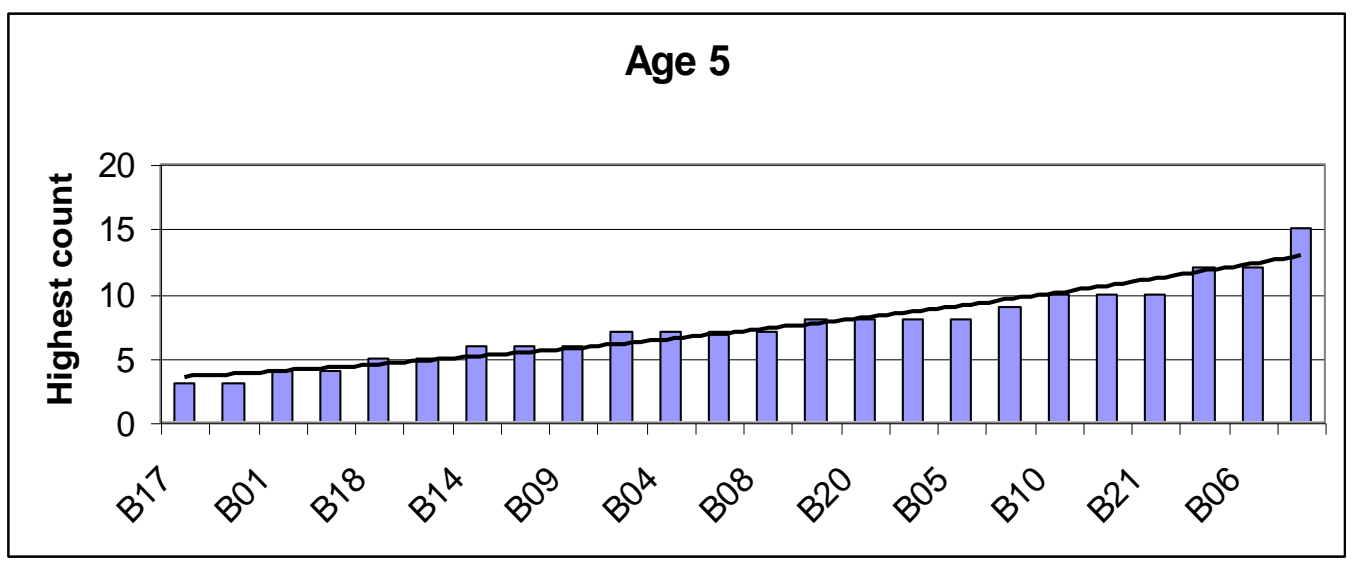

Figure 4: Five-year-olds' How many hidden game performances $(\mathrm{N}=24)$ 


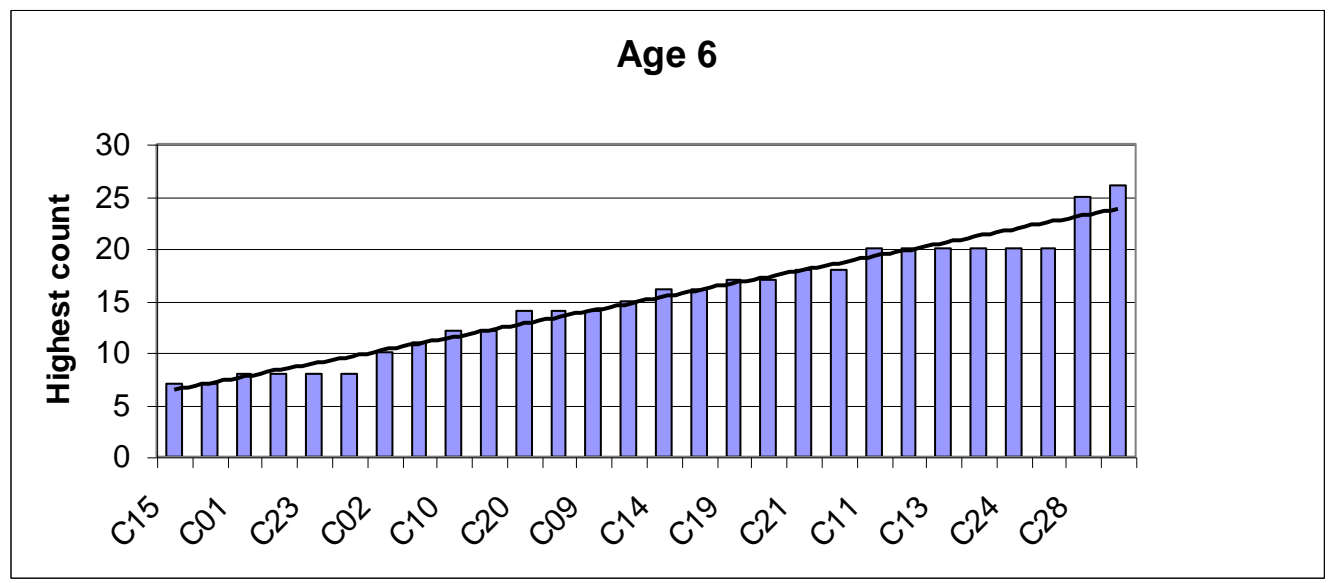

Figure 5: Six-year-olds' How many hidden game performances $(\mathrm{N}=\mathbf{2 8})$

work with quantities between 5 and 10 . The $3(12.5 \%)$ highest quantities, between 10 and 20, were unexpected of children so young and un-schooled. Note that this provides a warning to educators, that such a range, 3 to 20, may exist within their classes, and the needs of children at the extremes are very different. The Mean quantity for the 5-year-old group was 7.5, and the Standard Deviation 3.
Figure 5 shows that by age 6 , all children in the sample were working with quantities greater than 5, and most of them with quantities greater than 10 , with some beyond 15 or 20.

Seven $(25 \%)$ of the 6-year-olds were capable of working with quantities between 5 and 10 , while another 7 (25\%) were working within the range 11 to 15 . Unexpectedly, a further $12(42.9 \%)$ were working with quantities

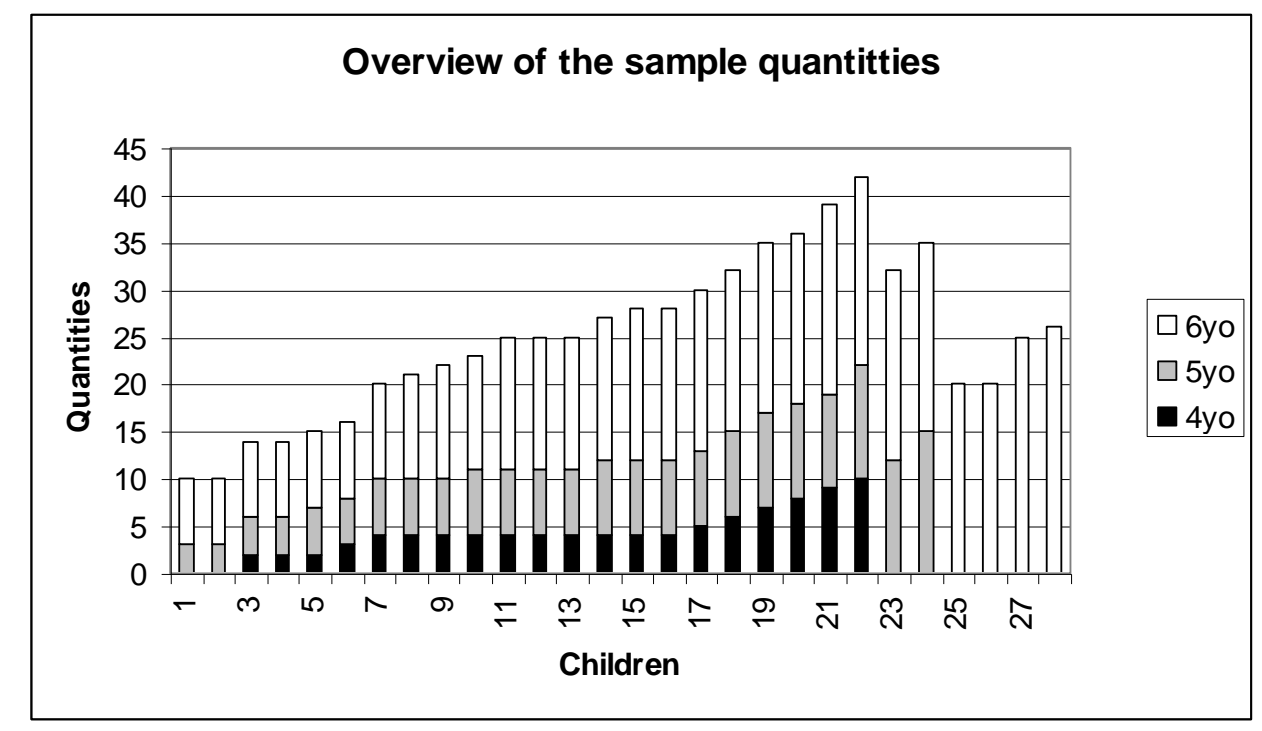

Figure 6: Composite of all How many hidden game performances $(\mathrm{N}=74)$ 
Connie Cassy Ompok, Brian Doig and Maria Tambagas

between 16 and 20 , and the last $2(7.1 \%)$ were working at 25 or 26 . The Mean quantity for the 6-year-olds was 15.04, and the Standard Deviation was 5.3.

Finally, Figure 6 shows all three groups represented on a single graph. Due to different numbers of children in each sample age group, the last six columns have no 4-yearold data, and the last 4 columns have only data from the 6-year-old sample.

\section{DISCUSSION}

While the small sample size may make generalizations difficult, the results demonstrate, clearly, that this approach to assessing young children's informal mathematical understanding is feasible and informative. While in this paper we have only described one of a range of assessment games, we believe that we have demonstrated that this approach is worth pursuing further. Assessment that describes development is a powerful tool to put into the hands of educators of young children.

\section{ACKNOWLEDGEMENTS}

We gratefully acknowledge the invaluable assistance from Early Childhood Students at
Universiti Malaysia Sabah (Rean Leh, Everina Emilly Tony, Sandra Julstero Loter and Beayzuanna Amylian Natasya Binti Badra) in conducting the interviews.

\section{REFERENCES}

Aunola, K., Leskinen, E., Lerkkanen, M. K., \& Nurmi, J. E. (2004). Developmental dynamics of math performance from preschool to grade 2. Journal of Educational Psychology, 96, 699-713.

Claessens, A., Duncan, G. J., \& Engel, M. (2009). Kindergarten skills and fifth grade achievement: Evidence from ECLS-K. Economics of Education Review 28(2009) 415-427.

Davydov, V. V. (1976). Psychological abilities of primary school children in learning mathematics. In L. P. Steffe (Ed.), Soviet studies in mathematics education (Vol. VI). Reston, VA: National Council of Teachers of Mathematics.

Romano, E., Babchishin, L., \& Pagani, L. S. (2010). School Readiness and Later Achievement: Replication and Extension Using a Nationwide Canadian Survey. Developmental Psychology, 46(5), 995-1007. 